\title{
UNE APPLICATION LUDIQUE DES ARCS ELECTRIQUES : LE TRANSFORMATEUR DE TESLA
}

\author{
G. Daveau, T. Martire, J.J. Huselstein, P. Enrici \\ Institut d'Electronique du Sud - Groupe et Energie Matériaux \\ - Université Montpellier II - \\ CC079 Place Eugène Bataillon, 34095 Montpellier cedex 05 \\ guillaume.daveau@univ-montp2.fr, martire@univ-montp2.fr, \\ huselstein@univ-montp2.fr, enrici@univ-montp2.fr
}

\begin{abstract}
Résumé :
L'objet de cet article est de mettre en avant la multitude d'applications liées de près ou de loin au arcs électriques. Nous nous attachons dans un premier temps à définir le phénomène. Nous présentons ensuite différentes applications. Enfin, nous mettons en oeuvre une maquette pédagogique permettant de réaliser un arc électrique. Cette maquette peut être réalisée au cours de projets de L3 ou de M1, mais elle peut également faire l'objet de TP de part le fonctionnement particulier du système de conversion d'énergie. L'attractivité de la maquette largement démontrée permet d'une part de sensibiliser les étudiants aux aspects plus théorique du fonctionnement du montage, et d'autre part, de mettre en avant l'importance et la place de la gestion d'énergie électrique vis à vis du grand public.
\end{abstract}

Mots clés : arcs électriques, transformateur de Tesla, hacheur, résonance

\section{Introduction}

Dans le cadre de l'enseignement du Master Sciences et Techniques Pour l'Ingénieur de l'Université de Montpellier II, nous avons mis oeuvre une séance de travaux pratiques concernant la génération d'arcs électriques liée au module applications modernes de l'énergie électrique.

Les objectifs sont de sensibiliser les étudiants aux différentes difficultés liées à la génération d'une haute tension.

Ainsi les étudiants font l'apprentissage de ce qu'est un arc électrique, et de la multitude d'application qui y sont liées tout en mettant en oeuvre une chaine de conversion d'énergie fonctionnant sur le principe d'un système résonnant.

Les élèves travaillent en binômes sur une séance de 4 heures et fournissent un compte-rendu.

Ils abordent ainsi :

- l'influence des capacités parasites des transformateurs haute tension,

- l'influence du couplage magnétique,

- le fonctionnement d'un hacheur résonnant.

\section{Historique}

Les arcs électriques sont des phénomènes naturels qui ont pu être observés depuis la nuit des temps : la foudre. Il a fallut attendre le cours de l'année 1813 pour que le premier arc électrique artificiel soit généré par le physicien anglais Humphrey Davy . Il venait d'ouvrir la porte vers un immense champ d'applications.
En effet, les ingénieurs ont rapidement compris le parti qu'ils pouvaient tirer d'un tel phénomène. Ainsi, dés 1880, les trois propriétés fondamentales de l'arc électrique étaient découverte et mises en application :

- flux radiatif intense (Lampe à arc à haut flux, Léon Foucault, 1844);

- possibilité de favoriser les réactions chimiques (Synthèse de l'acétylène en atmosphère d'hydrogène, Marcellin Berthelot, 1862) ;

- flux thermique élevé (Soudure à l'arc, Louis Clerc, 1880).

En 1919 Ernest Rutherford transforma des atomes d'azote en oxygène en les bombardant avec des particules alpha générées par un isotope radioactif. La transmutation des éléments, le domaine mythique des alchimistes était, au moins à l'échelle microscopique, en train de devenir une réalité. $\mathrm{La}$ désintégration de l'atome nécessitant de très hautes énergies, une première solution possible était d'accélérer les particules dans un tube à vide soumis à une très haute tension. La course au million de volts avait commencé. L'un des concurrent était Nicolas Tesla qui présenta un transformateur générant des arcs électriques spectaculaires d'une dizaine de mètres pour l'exposition universelle de Chicago en 1893. On citera également Robert Jamison Van de Graaff, qui choisit, à la même époque, de développer une machine électrostatique.

L'apparition des théories modernes de la physique alliées aux maitrises de conceptions industrielles 
vont permettre peu à peu la mise en oeuvre de démarches scientifiques conduisant à une meilleure connaissance des propriétés fondamentales de l'arc électrique laissant place à l'empirisme qui était la règle.

On citera La Loi de Paschen, énoncée par le physicien allemand Friedrich Paschen en 1889, indiquant que l'apparition d'un arc électrique dans un gaz, à un certain champ électrique de claquage (dit champ disruptif). Cette fonction étant généralement non linéaire du produit de la pression du gaz par la distance entre les électrodes.

Ces dernières décennies, l'arc électrique a fait l'objet de nombreuses études fondamentales en étroite collaboration avec les organismes industriels concernés. On trouve, dans la littérature, des définitions très différentes de l'arc électrique. On admet généralement que le nom d'arc électrique est associé à une décharge à forte densité de courant pour laquelle les processus thermiques sont dominants [1].

\section{Définition}

Un arc est une décharge électrique entre deux électrodes auto-entretenue, ayant une faible chute de tension aux électrodes et capable de supporter de forts courants.

Un arc se crée entre deux surfaces de conducteurs par une ionisation du gaz lorsque la différence de potentiel entre les deux électrodes est suffisamment élevée.

On défini le champ disruptif du gaz exprimé en $\mathrm{V} / \mathrm{m}$. L'air présente par exemple des champs disruptifs compris entre $1 \mathrm{kV} / \mathrm{mm}$ et $6 \mathrm{kV} / \mathrm{mm}$ en fonction de la pression atmosphérique et de l'humidité ambiante.

Une fois ionisé, le gaz crée un canal conducteur qui entraine un arc continue tant que la différence de potentiel reste suffisante.

En théorie, la position de l'arc électrique est stable (principe de l'énergie minimale). Toute fois, nous verrons par la suite que cette caractéristique reste discutable.

L'écoulement du courant dans la matière ionisée émet un rayonnement de lumière dont le spectre est caractéristique de la nature du gaz, et à un degré moindre, de celle des électrodes.

Cette ionisation et l'écoulement d'un courant électrique qui s'en suit engendrent également des bruits dus à l'expansion brutale du gaz suite à son échauffement tout aussi brutal (propriété également exploitée dans diverses applications) [2].

\section{Applications des arcs électriques}

Les applications liées de prés ou de loin aux arcs électriques sont nombreuses. Certaines sont (re)connues et industrialisées depuis longtemps, d'autres ne sont encore que des idées ou projets scientifiques. Il existe un très grand nombre d'applications et d'études des arcs électriques portant sur la fiabilité de systèmes et leurs comportements face aux décharges électriques (disjoncteurs, EDF, avionique, etc...) [3], mais également des études concernant les phénomènes d'éclairs naturels en météorologie, médecine, et également en électronique de puissance pour les aspects CEM [4]. Nous allons brièvement présenter certaines de ces applications.

\subsection{Lampe à arc}

Les lampes à arc créent de la lumière en faisant jaillir une étincelle qui passe d'une électrode à l'autre. Cette lumière brille bien plus que toutes les sources d'illumination jamais inventées auparavant.

Aujourd'hui, le développement et la fabrication de lampes à décharge d'arc sont destinées à des applications industrielles précises telles que le pompage lasers, la photographie moyenne et forte puissance, la médecine, les simulations solaire, les feux anti-collision pour l'aéronautique...

On retrouve également les lampes à arc dans le domaine grand public pour des applications d'éclairage permettant d'atteindre de haut rendement. Les lampes à sodium (éclairage urbain jaunâtre), les lampes à iodures métalliques (éclairage urbain banc), les lampes fluorescentes et basse consommation (éclairage intérieur), les lampes aux halogénures métalliques (éclairage blanc des stades), les lampes au Xénon (certains phares d'automobile, les projecteurs de cinéma).

\subsection{Four à arc}

Le premier à avoir mis en avant l'application des arc électriques pour le chauffage à haute température est Henri Moissan en 1892 réalisant un four permettant d'atteindre jusqu'à $3500^{\circ} \mathrm{C}$. Il isolera ainsi plusieurs métaux et mettra au point la fabrication de plusieurs composés tels que les carbures [2].

$\mathrm{Au}$ début du $\mathrm{XX}^{\text {ième }}$ siècle sont apparus les premiers fours à arc industriels destiné à fondre un métal primaire (généralement ferraille) pour obtenir un acier. Depuis cette époque, bien que reposant sur les mêmes principes de base, les fours à arc ont connu un développement important aussi bien du point de vue technologique que du point de vue capacité de production. En effet, la capacité des fours modernes avoisine les 200T avec une consommation énergétique comprise entre 400 et $500 \mathrm{kWh} / \mathrm{t}$.

\subsection{Soudure à arc}

Le soudage à l'arc électrique est le plus connu des procédés de soudure. Il existe différentes méthodes de soudage électrique (électrodes enrobées, électrode non-fusible, électrode fusible). La plus utilisée est le soudage à arc électrique avec électrode enrobée. Lorsqu'on approche l'électrode enrobée des 
pièces à assembler, il se crée un arc électrique qui dégage un fort pouvoir calorique qui provoque la fusion de l'électrode [2].

\subsection{Bougies d'allumage}

La bougie d'allumage est l'élément qui crée l'étincèle dans le cylindre pour permettre l'explosion du carburant dans le moteur dit "à explosion" (utilisé dans nos motos et voitures actuelles).

Au du siècle, une même bougie pouvait être utilisé pour tous types de véhicules, car il suffisait à l'époque de faire une étincelle, sans se soucier de ses caractéristiques ni des problème liés à la dissipation des calories du moteur.

\subsection{Appareils de coupure}

La coupure d'un courant électrique est obtenue en séparant des contacts dans un gaz (air, $\mathrm{SF}_{6}, \ldots$ ) ou dans un milieu isolant. Après séparation des contacts, le courant continue de circuler dans le circuit à travers un arc électrique qui s'établit entre les contacts. On cherche alors à maitriser et éteindre l'arc le plus rapidement possible.

Pour ce faire, on peut par exemple "souffler" l'arc à l'aide d'un gaz sous pression. L'arc crée lors de l'ouverture du disjoncteur est "soufflé" par un gaz sous pression présentant de bonnes caractéristiques diélectriques (comme le $\mathrm{SF}_{6}$ ).

\subsection{Torche à plasma}

Une torche à plasma est un dispositif permettant d'atteindre de très hautes températures : $1600^{\circ} \mathrm{C}$. Sa conception trouve son origine dans l'étude de matériaux destinés à protéger les missiles ou les engins spatiaux durant leur traversée de l'atmosphère [2].

Aujourd'hui, elles sont utilisées comme une solution de recyclage pour les déchets dangereux (résidus d'incinération, amiante, déchets faiblement radioactifs, poussières, déchets industriels, ...) afin d'éviter l'enfouissement en décharge et le recyclage des "déchets ultimes" (déchets de déchets)[5].

Pour générer le plasma, on met en contact le gaz avec un arc électrique. Cet arc étant amorcé entre deux électrodes (cathode et anode) : c'est le principe de l'arc soufflé.

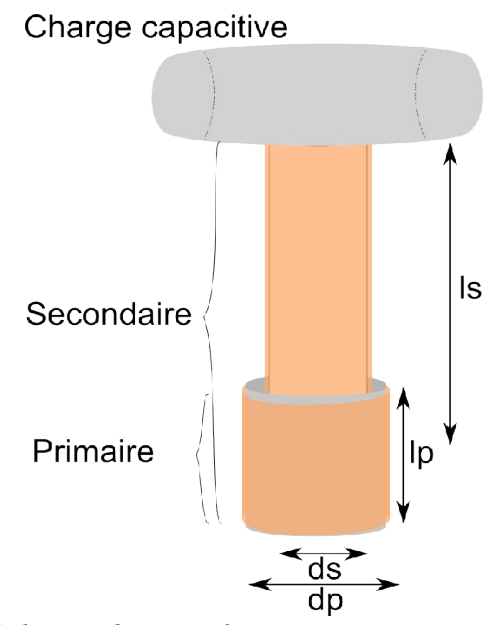

Fig 1: Schéma du transformateur

\subsection{Projets et recherche sur les plasmas}

Les recherches sur les plasmas ont démarrer avec l'étude de la foudre (c'était l'objectif de Davy...) et ensuite elles ont prit de l'ampleur grâce aux nombreuses applications industrielles.

\section{Le transformateur de Tesla}

L'application étudiée dans le TP est appelée transformateur de Tesla. Le schéma du dispositif est donné figure 1 .

Nous avons choisi de présenter ce système car il est simple à mettre en oeuvre pour un cout très faible. D'un point de vue pédagogique, les manipulations et mesures pouvant êtres effectuées sur le dispositifs sont aussi nombreuses que les applications présentées. Enfin, le pouvoir d'attraction de ce type de maquettes pédagogique est également non négligeable.

Le schéma électrique du dispositif est présenté figure 2. La capacité parallèle située au secondaire du transformateur est constituée d'une part par la charge capacitive présenté sur le schéma figure 1, et d'autre part par la capacité entre les spires du secondaire et la terre.

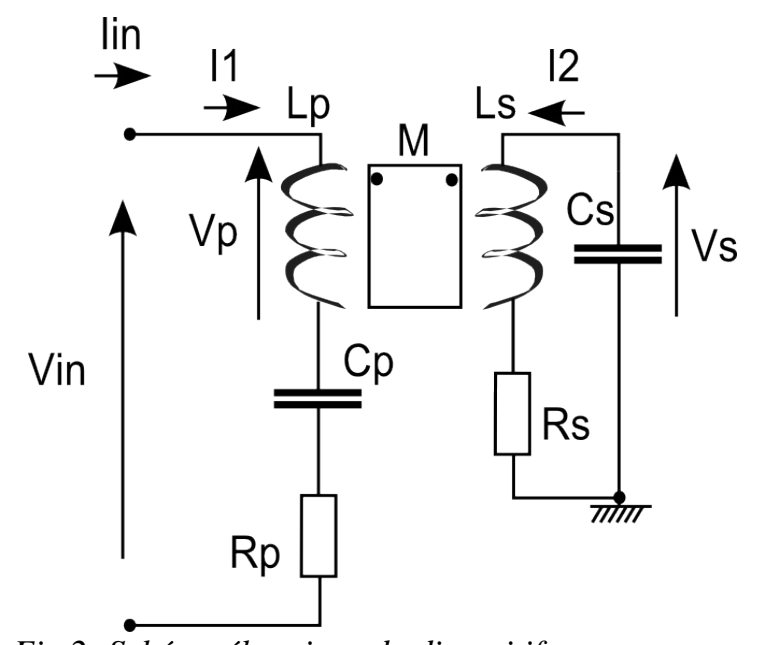

Fig 2: Schéma électrique du dispositif

Pour mieux appréhender le comportement du dispositif, il faut exprimer les fonctions de transfert en tension. Pour simplifier les expressions, on néglige les résistances, et on exprime :

$$
w_{p}=\frac{1}{\sqrt{L_{p} C_{p}}}
$$

et :

$$
w_{s}=\frac{1}{\sqrt{L_{s} C_{s}}}
$$

On peut alors exprimer la fonction de transfert en tension :

$$
T v=\frac{C_{p} M w^{2}}{1-C_{p} C_{s} M^{2} w^{4}-C_{p} C_{s} L_{s} L_{p} w^{4}-\left(C_{s} L_{s}+C_{p} L_{p}\right) w^{2}}
$$


Considérons un coefficient de couplage $\mathrm{k}$ :

$$
M=k \sqrt{L_{p} L_{s}}
$$

En simplifiant, on obtient :

$$
T v=\frac{C_{p} M w^{2}}{1-\frac{\left(1-k^{2}\right) w^{4}}{\left(w_{p} w_{s}\right)^{2}}-\frac{\left(w_{p}^{2}+w_{s}^{2}\right) w^{2}}{\left(w_{p} w_{s}\right)^{2}}}
$$

On retrouve alors les valeurs des fréquences de résonances du dispositif [6] :

$$
w_{1}=\sqrt{w_{s}^{2}+w_{p}^{2}-\frac{\sqrt{\left(w_{s}^{2}-w_{p}^{2}\right)^{2}+4 k^{2}\left(w_{s} w_{p}\right)^{2}}}{2\left(1-k^{2}\right)}}
$$

et :

$$
w_{2}=\sqrt{w_{s}^{2}+w_{p}^{2}+\frac{\sqrt{\left(w_{s}^{2}-w_{p}^{2}\right)^{2}+4 k^{2}\left(w_{s} \cdot w_{p}\right)^{2}}}{2\left(1-k^{2}\right)}}
$$

On notera le fait que ces fréquences de résonances dépendent de la valeur du coefficient de couplage des inductances. La figure 3 présente les fonctions de transfert en tension et impédance d'entrée simulées à l'aide d'un programme matlab ceci pour les valeurs mesurées des éléments.

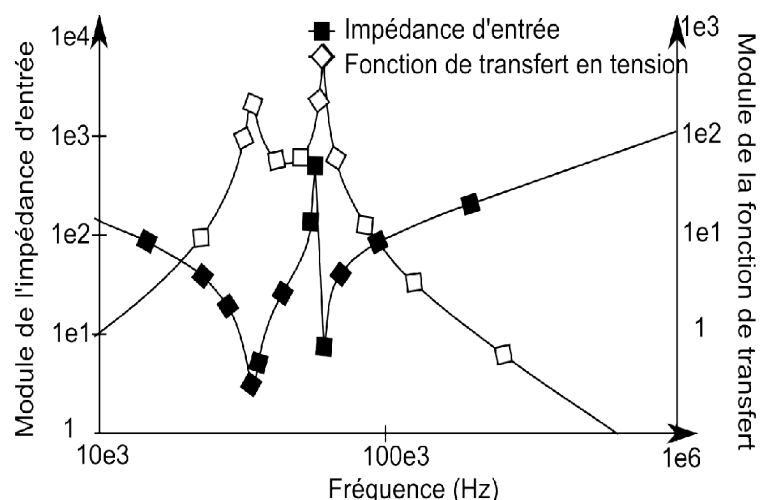

Fig 3: Impédance d'entrée et fonction de transfert en tension simulées

\section{Déroulement de la scéance de TP}

La partie commande et la partie puissance sont des démonstrateurs pédagogiques permettant un fonctionnement du système selon différents modes (hacheur, onduleur...). La commande, entièrement numérique, s'organise autour d'un FPGA. La partie puissance est composée par trois bras d'onduleur, respectivement formées par deux IGBT. On peut également modifier la fréquence de découpage ainsi que le rapport cyclique simplement à l'aide de codeurs incrémentaux [7]. On utilisera une commande de type hacheur série avec une commande simultanée sur les trois bras de l'onduleur afin d'augmenter le courant maximal du convertisseur.

6.1 Mesure des valeurs des éléments inductifs

Les valeurs des éléments inductifs Lp et Ls sont mesurés en basse fréquence au multimètre numérique avec un système découplé (primaire et secondaire éloignés). Les valeurs de ces éléments pouvant être comparés aux valeurs théoriques attendues définies par la géométrie du bobinage. On a ainsi pour l'inductance primaire :

$$
\begin{gathered}
N_{p}=20 \text { spires } \\
l_{p}=5 \mathrm{~cm} \text { (longueur du bobinage) } \\
d_{p}=30 \mathrm{~cm} \text { (diamètre du bobinage) } \\
L_{p}=\frac{N_{p}^{2}}{\frac{2 l_{p}+d_{p}}{2} \mu_{0} \pi \frac{d_{p}^{2}}{4}}=177 \mu \mathrm{H} \\
L_{p_{-}} \text {mesurée }=199 \mu \mathrm{H}
\end{gathered}
$$

De même pour l'inductance du secondaire :

$$
\begin{gathered}
N_{s}=26 \text { spires } / \mathrm{cm} \text { avec } 63 \mathrm{~cm} \text { soit } 1638 \text { spires } \\
l_{s}=64 \mathrm{~cm} \text { (longueur bobinage) } \\
d_{s}=25 \mathrm{~cm} \text { (diamètre du bobinage) } \\
L_{s}=\frac{N_{s}^{2}}{\frac{2 l_{s}+d_{s}}{2} \mu_{0} \pi \frac{d_{s}^{2}}{4}}=216 \mathrm{mH} \\
L_{s \_} \text {mesurée }=257 \mathrm{mH}
\end{gathered}
$$

On remarque ici que les valeurs calculées à partir des modèles classiques des éléments inductifs sont relativement proches des valeurs réelles mesurées.

\subsection{Mesure des fréquences de résonances propres}

Pour mesurer indépendamment les fréquences de résonance de chaque bobine, nous allons découpler les deux inductances en les éloignant. On mesure ainsi la fréquence de résonance au primaire simplement :

$$
f_{p}=36,3 \mathrm{kHz}
$$

On peut comparer cette valeur à la valeur théorique connaissant la valeur de la capacité résonnante :

$$
C_{p}=0,1 \mu F
$$

Soit :

$$
f_{p}=35,6 \mathrm{kHz}
$$

Pour la mesure de la fréquence de résonance propre du secondaire, on va l'exciter avec le primaire et mesurer le courant dans le secondaire avec un coefficient de couplage proche de zéro. On mesure ainsi une fréquence de résonance propre de :

$$
f_{s}=57 \mathrm{kHz}
$$

On en déduit la valeur de la capacité parallèle équivalente au secondaire :

$$
C_{s}=30 p F
$$

\subsection{Calcul du coefficient de couplage}

Pour retrouver la valeur du coefficient de couplage, on solidarise les inductances primaires et secondaire et on mesure les fréquences de résonance. On obtient alors :

$$
F_{p}=34 \mathrm{kHz}
$$


et :

$$
F_{s}=60 \mathrm{kHz}
$$

On en déduit la valeur du coefficient de couplage :

$$
k=30 \%
$$

6.4 Calcul de la tension approximative aux bornes du secondaire

Une fois la fréquence de résonance correctement choisie, on peut mesurer la valeur du courant dans le secondaire qui nous renseigne sur la valeur approximative de la tension de sortie aux bornes du secondaire $\mathrm{du}$ transformateur. Les données électriques permettant d'obtenir un bon fonctionnement du transformateur sont rappelées dans le tableau 1.

\begin{tabular}{|c|c|}
\hline $\mathrm{F}$ & $60 \mathrm{kHz}$ \\
\hline Ve (DC) & $300 \mathrm{~V}$ \\
\hline Ip (eff) & $9 \mathrm{~A}$ \\
\hline Is (eff) & $420 \mathrm{~mA}$ \\
\hline Vp (eff) & $680 \mathrm{~V}$ \\
\hline Vs (eff) & $40 \mathrm{kV}$ \\
\hline longueur & $15 \mathrm{~cm}$ \\
\hline
\end{tabular}

Tableau 1: Résumé des mesures

\section{Résultats en image}

On constate l'apparition d'arcs électriques.
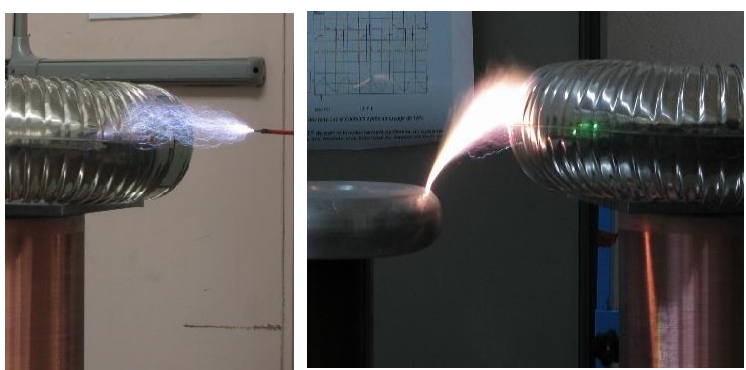

Fig 4: Photographies de l'arc état instable à gauche et stable à droite

On remarque également l'influence du champ électrique sur l'environnement ambiant comme le démontre la photographie 4, ou le tube fluorescent s'allume sans apport d'énergie extérieure.

\section{Conclusions}

Les arcs électriques sont aujourd'hui présents dans de nombreuses applications allant de l'utilisation quotidienne à l'industrie haute technologie. La mise en oeuvre de ce TP d'électronique de puissance mettant en avant ces phénomène va donc de soit.

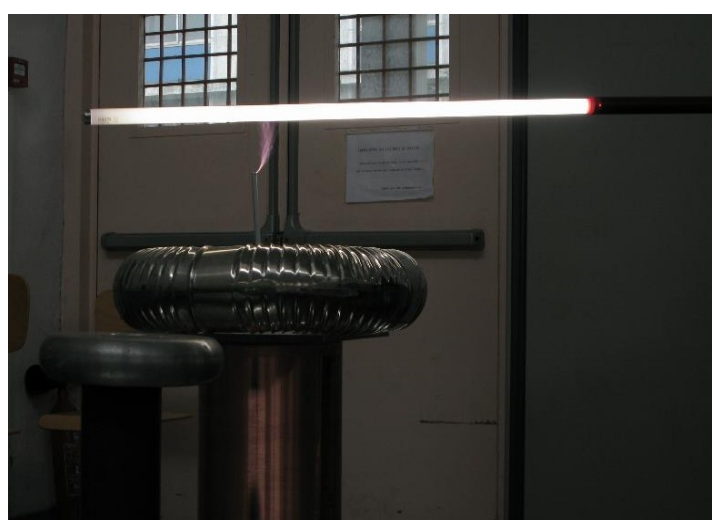

Fig 5: Tube fluorescent sans fil

Le choix du transformateur de Tesla se justifie par différents aspects. D'une part, le fonctionnement du dispositif est très attractif non seulement vis à vis des étudiants, mais également vis à vis du grand public. D'autre part, le fonctionnement particulier du transformateur permet de mettre en avant les nuisances des paramètres parasites généralement négligés ainsi que l'intérêt des systèmes résonants. Enfin, la simplicité du dispositif de commande et de puissance permettent une approche intuitive facilitant la mise en oeuvre et l'étude du dispositif.

\section{Bibliographie}

1. Serge Vacquie, Arc électrique, Techniques de l'ingénieur, Génie électrique,1995, vol. D4, n'D2870, pp. 1-28

\section{2. http://fr.wikipedia.org/}

3. Claude Gary, L'arc électrique et ses applications, Revue de Génie Electrique, 1983, vol. 4, pp. 264-5

4. J. L. Cocquerelle C.E.M. et électronique de puissance, édition Technip, 1999

5. http://www.europlasma.com/

6. Marco Denicolai, High Voltage Institute, Helsinki University of Technology, P.O. Box 3000, FIN 02015 HUT, Finland

7. J.J. Huselstein, T. Martiré, Ensemble pédagogique expérimental hacheur onduleur, CETSIS, Nancy, Octobre 2005 